\title{
STANDARD MODELS OF SET THEORY WITH PREDICATION
}

\author{
BY THOMAS J. JECH ${ }^{1}$ AND WILLIAM C. POWELL ${ }^{2}$
}

Communicated by Dana Scott, April 15, 1971

1. Introduction. We consider another axiomatization of set theory. It is a first-order theory with equality, the membership relation, a new binary relation called predication and denoted by backwards epsilon, and a constant $V$ denoting the universe of sets. Sets are defined to be elements of $V$. Classes are defined to be collections of sets. The variables $P, Q, R$ are defined to range over classes. Thus, $\forall P \Phi(P)$ is short for

$$
\forall x(\forall y(y \in x \rightarrow y \in V) \rightarrow \Phi(x)) .
$$

We only consider classes on the left of predication. The axioms are the universal closures of

(A) $x \in y \in V \rightarrow x \in V$,

(B) $\forall x \in V(P \ni x \leftrightarrow x \in P)$,

(C) $\forall x \in V(P \ni x \leftrightarrow Q \ni x) \rightarrow P=Q$,

(D) $\forall x_{1}, \cdots, x_{n} \in V \exists Q \forall y\left(Q \ni y \leftrightarrow \Phi\left(P_{1}, \cdots, P_{n} ; x_{1}, \cdots, y_{1}\right)\right)$, where $\Phi$ is a formula such that

(i) all its free variables are displayed,

(ii) the $P$ 's are the only variables occurring on the left in predication,

(iii) the $P$ 's occur only on the left in predication, and

(iv) $V$ does not occur.

We also add as axioms that

(E) $V$ satisfies the axiom of choice and

(F) $V$ satisfies the axiom of regularity.

This theory was first considered by the second author and will be presented in his thesis along with the proofs of the following theorems in the theory.

AMS 1970 subject classifications. Primary 02K15, 02K35; Secondary 02K10.

Key words and phrases. Axiomatization of set theory, predication, large cardinals, indescribable cardinals, ineffable cardinals, standard models, $M$-ultrafilters, wellfounded ultrapowers.

1 The first author acknowledges support received under NSF Grant GP-22937.

2 The second author acknowledges support through a NSF Traineeship. 


$$
\begin{aligned}
\vdash \forall x_{1}, \cdots, x_{n} \in V\left(\left(V \vDash \Phi\left(P_{1}, \cdots, P_{m} ; x_{1}, \cdots, x_{n}\right)\right) \leftrightarrow\right. \\
\left.\Phi\left(P_{1}, \cdots, P_{m} ; x_{1}, \cdots, x_{n}\right)\right)
\end{aligned}
$$

where $\Phi$ is a formula satisfying (i)-(iv) of axiom (D), and $V \models \Phi$ is the relativization of $\Phi$ to $V$. In a strong sense (1.0) says $V$ is an elementary submodel of the universe of discourse.

$$
\vdash \mathrm{ZFC} \text {. }
$$

That is, all the axioms of Zermelo-Fraenkel set theory plus the axiom of choice are derivable. Actually the proofs of the axioms of ZFC yield slightly stronger results. For example,

$$
\vdash \exists y \forall z\left(z \in y \leftrightarrow z \in x \wedge \Phi\left(P_{1}, \cdots, P_{m} ; w_{1}, \cdots, w_{n}, z\right)\right),
$$

where $\Phi$ is a formula satisfying (i)-(iv) of axiom (D), is the version of the comprehension principle that is derived. Since $V$ is the class of all sets, it should be noted that by (1.0) and (1.1) that we also have

$$
\vdash V \vDash \mathrm{ZFC} \text {. }
$$

We also have the following reflection principle for classes

$$
\vdash Q \ni P \rightarrow \exists x \in V(Q \ni P \cap x) \text {. }
$$

In this paper we will show first that the existence of large cardinals is derivable in the theory. Although (1.4) essentially is a large cardinal property, it is formulated in terms of predication. We will deduce two large cardinal properties formulated only in terms of $\in$. If $\kappa$ is the class of ordinals in $V$, then

THEOREM 1. $\leftarrow \kappa$ is indescribable.

Theorem 2. $\vdash \kappa$ is ineffable.

Both of these properties imply the existence of weakly compact cardinals.

Secondly we will give a necessary and sufficient condition for standard models of the theory to exist.

THEOREM 3. There exists a standard model of the theory (A)-(F) if and only if there exists a standard model $M$ of $Z F C$ and an $M$-ultrafilter $U$ on some ordinal $\kappa$ in $M$ such that $\operatorname{Ult}(\kappa, U)$ is wellfounded.

This condition follows from the existence of measurable cardinals. It even follows from some weaker properties. $R$. Solovay pointed out that it follows from J. Silver's work [3] that $\exists \kappa \kappa \rightarrow(\omega)<\omega$ implies the condition. 


\section{Large cardinals.}

Definition. A cardinal $\kappa$ is said to be indescribable if for any $U \subseteq R_{\kappa}$, any $n, m \in \omega$, and any $\Pi_{m}^{n}$ sentence $\sigma$ holding in $\left\langle R_{\kappa}, \in, U\right\rangle$, there exists an ordinal $\alpha<\kappa$ such that $\sigma$ holds in $\left\langle R_{\alpha}, \in, U \cap R_{\alpha}\right\rangle$.

Theorem 1. $\vdash \kappa$ is indescribable.

Proof. Suppose $U \subseteq R_{\kappa}$ and $\sigma$ is a $\Pi_{m}^{n}$ sentence holding in $\left\langle R_{\kappa}, \in, U\right\rangle$. By axiom (D) there exists $Q$ such that $Q \ni y$ if and only if dom $y$ is an ordinal,

$$
\forall z\left(U \ni x \wedge x \in R_{\text {dom } y} \leftrightarrow x \in \operatorname{rg} y\right),
$$

and $\sigma$ holds in $\left\langle R_{\operatorname{dom} y}, \in \operatorname{rg} y\right\rangle$. If we let $P=\kappa \times U$, then we have $Q \ni P$. Hence by (1.4), there exists some $x \in V$ such that $Q \ni P \cap x$. Hence, by axiom (B), $\alpha=\operatorname{dom}(P \cap x)$ is an ordinal, $\operatorname{rg}(P \cap x)=U \cap R_{\alpha}$, and $\sigma$ holds in $\left\langle R_{\alpha}, \in, U \cap R_{\alpha}\right\rangle$.

Now we introduce some suggestive, though unnecessary, notation. Instead of writing $P \ni x$ we will write $x \in j(P)$. Although formally " $\in j()$ " is an indivisible relation symbol it is useful to think of " $j(P)$ " as the extension $\{x: P \ni x\}$ of $P$. In general the extension of a class will not exist in the universe of discourse. $\{x: P \ni x\}$ exists if and only if $P$ is a set. And if $P$ is a set, $P=j(P)$.

If $f \subseteq V$ is a function from $P$ to $Q$, then $j(f)$ can be thought of as a function from $j(P)$ to $j(Q)$. This situation is precisely expressed by the equivalence of the following formulas:

$$
\begin{array}{cll}
\forall x \in P & \exists ! y \in Q & \langle x, y\rangle \in f, \\
V \vDash \forall x \in j(P) & \exists ! y \in j(Q) & \langle x, y\rangle \in j(f), \\
\forall x \in j(P) & \exists ! y \in j(Q) & \langle x, y\rangle \in j(f) .
\end{array}
$$

We will denote the unique $y$ in the last formula by $j(f)(x)$.

Definition. $A$ is a closed subset of $\kappa$ if $\forall \alpha<\kappa \forall x \subseteq A \cup(\alpha \cap x) \in A$. $A$ is an unbounded subset of $\kappa$ if $\bigcup A=\kappa . S \subseteq \kappa$ is said to be stationary if $S$ intersects every closed unbounded subset of $\kappa$.

The following notion is due to R. Jensen [1].

Definition. $\kappa$ is ineffable if for every $f: \kappa \rightarrow P(\kappa)$ such that $\forall \alpha<\kappa$ $f(\alpha) \subseteq \alpha$, there exists $X, S \subseteq \kappa$ such that $S$ is stationary and $\forall \alpha \in S$ $f(\alpha)=X \cap \alpha$.

\section{Theorem 2. $\kappa$ is ineffable.}

Proof. Suppose $f$ is a function on $\kappa$ such that $\forall \alpha<\kappa f(\alpha) \subseteq \alpha$. Then since $\forall \alpha<\kappa f(\alpha) \in V$, we have $f \subseteq V$. Since

$$
\forall x(\kappa \ni x \leftrightarrow x \text { is an ordinal), }
$$


$\kappa \in j(\kappa)$. Hence, we may take $X=j(f)(\kappa)$. By $(1.0), j(f)(\kappa) \subseteq \kappa \subseteq V$. Hence, by axiom (D), there exists $S$ such that

$\left(^{*}\right) \quad S \ni \alpha \leftrightarrow \exists y(f \ni\langle\alpha, y\rangle \wedge \forall z(z \in y \leftrightarrow j(f)(\kappa) \ni z \wedge z \in \alpha))$.

Hence, by axiom (B), for $\alpha \in \kappa$,

$$
\alpha \in S \leftrightarrow f(\alpha)=j(f)(\kappa) \cap \alpha .
$$

Now to show that $S$ is stationary, suppose $A$ is a closed and unbounded subset of $\kappa$. Then by axiom (B) and (1.0) we have

$$
\forall z\left(\forall y(y \in z \rightarrow A \ni y) \rightarrow A \ni \bigcup_{z}\right) .
$$

Hence, since $\forall y(y \in A \rightarrow A \ni y)$ and $\bigcup A=\kappa$, we have $A \ni \kappa$. And since by $\left({ }^{*}\right), S \ni \kappa$, we have

$$
\exists z(S \ni z \wedge A \ni z) .
$$

Thus, by (1.0)

$$
\exists z \in V(S \ni z \wedge A \ni z) .
$$

So by axiom (B), $S$ intersects $A$.

Although we have not actually shown any sets are indescribable or ineffable, it can be easily deduced from (1.0) that there are many sets that are indescribable and ineffable.

3. Standard models. The only models we consider in this paper are standard. Thus, a model of ZFC is a transitive model where the epsilon symbol is interpreted as the usual membership relation. And a model of $(\mathrm{A})-(\mathrm{F})$ is a triple $\left\langle M, V^{*}, \ni^{*}\right\rangle$ where $M$ is transitive, $V^{*}$ is the interpretation of $V, \ni^{*}$ is the interpretation of predication, and the interpretation of the epsilon symbol is understood to be the usual membership relation.

Definition. If $M$ is a model of $Z F C$ and $\kappa$ is an ordinal in $M$, then $U$ is an $M$-ultrafilter if and only if $\kappa>\omega$ and

(i) $U$ is a proper subset of $P(\kappa) \cap M$ containing no singletons,

(ii) $\forall x, y(x \subseteq y \in P(\kappa) \cap M \wedge x \in U \rightarrow y \in U)$,

(iii) $\forall x \in P(\kappa) \cap M(x \in U \bigvee \kappa-x \in U)$,

(iv) if $\eta<\kappa,\left\langle x_{\xi}: \xi<\eta\right\rangle \in M$ and $\forall \xi<\eta x_{\xi} \in U$, then $\cap\left\{x_{\xi}: \xi<\eta\right\} \in U$,

(v) if $\left\langle x_{\xi}: \xi<\kappa\right\rangle \in M$, then $\left\{\xi: x_{\xi} \in U\right\} \in M$.

This notion and the definition of the ultrapower Ult $(M, U)$ of $M$ with respect to an $M$-ultrafilter $U$ is due to K. Kunen [2].

TheOREM 3. There exists a standard model of the theory $(\mathrm{A})-(\mathrm{F})$ if and only if there exists a standard model $M$ of $Z F C$ and an $M$-ultrafilter 
$U$ on some ordinal $\kappa$ in $M$ such that $\operatorname{Ult}(\kappa, U)$ is wellfounded.

Proof. Necessity. Suppose $\left\langle M, V^{*}, \ni^{*}\right\rangle \vDash(\mathrm{A})-(\mathrm{F})$. Then it suffices to show that $M$ is a model of ZFC, $U=\{P \subseteq \kappa: \kappa \in j(P)\}$ is an $M$ ultrafilter on $\kappa$, where $\kappa$ is the class of ordinals in $V^{*}$, and Ult $(\kappa, U)$ is wellfounded. By (1.1), $M$ is a model of ZFC. We now show that $U$ is an $M$-ultrafilter. It is easily seen that $\kappa>\omega$ and (i)-(iii) hold. (iv) follows from

$$
\begin{aligned}
& \vdash \forall x \in V \forall f: x \rightarrow P(V) \forall y \\
& \qquad(\bigcap\{f(z): z \in x\} \ni y \leftrightarrow \forall z \in x f(z) \ni y) .
\end{aligned}
$$

To see $\left(^{* *}\right)$ let $P=\left\{\langle y, z\rangle \in V: y \in F_{z}\right\}$. Then, for all $z \in V$,

$$
\forall y(P \ni\langle y, z\rangle \leftrightarrow f(z) \ni y) \text {. }
$$

By axiom (D) we have some $Q$ such that

$$
\forall y(Q \ni y \leftrightarrow \forall z \in x P \ni\langle y, z\rangle) .
$$

Hence,

$$
\forall y(Q \ni y \leftrightarrow \forall z \in x f(z) \ni y) .
$$

But $Q=\bigcap\{f(z): z \in x\}$ by axiom (B). To show (v) suppose $x: \kappa \rightarrow P(\kappa)$ is in $M$. Then $P=\left\{\langle\xi, \eta\rangle: \eta \in x_{\xi}\right\}$ is in $M$. Then for all $\xi<\kappa$,

$$
\forall \eta\left(P \ni\langle\xi, \eta\rangle \leftrightarrow x_{\xi} \ni \eta\right) .
$$

Hence, by (1.2), $\left\{\xi \in \kappa: x_{\xi} \in U\right\}=\left\{\xi \in \kappa: x_{\xi} \ni \kappa\right\}=\{\xi \in \kappa: P \ni\langle\xi, \kappa\rangle\}$ is in $M$.

To show that $\operatorname{Ult}(\kappa, U)$ is wellfounded, it suffices to construct an $\in$-isomorphism $k: \mathrm{Ult}(\kappa, U) \rightarrow M$, since $M$ is wellfounded. Let $k$ be defined by $k([f])=j(f)(\kappa)$ where $f: \kappa \rightarrow \kappa$. $k$ is well-defined and one-toone since

$$
\begin{aligned}
{[f]=[g] } & \leftrightarrow\{\xi \in \kappa: f(\xi)=g(\xi)\} \in U \leftrightarrow \kappa \in j\{\xi \in \kappa: f(\xi)=g(\xi)\} \\
& \leftrightarrow j(f)(\kappa)=j(g)(\kappa) .
\end{aligned}
$$

$k$ is an $\in$-isomorphism since

$$
\begin{aligned}
{[f] \in[g] } & \leftrightarrow\{\xi \in \kappa: f(\xi) \in g(\xi)\} \in U \leftrightarrow \kappa \in j\{\xi \in \kappa: f(\xi) \in g(\xi)\} \\
& \leftrightarrow j(f)(\kappa) \in j(g)(\kappa) .
\end{aligned}
$$

Sufficiency. Now suppose $M$ is a model of ZFC, $U$ is an $M$-ultrafilter on an ordinal in $M$, and Ult $(\kappa, U)$ is wellfounded. Since Ult $(\kappa, U)$ is wellfounded, $\operatorname{Ult}\left(P^{M}\left(R_{\kappa} \cap M\right), U\right)$ is wellfounded, and hence can be identified with some transitive class. Let $i: P^{M}\left(R_{\kappa} \cap M\right) \rightarrow$ 
$\operatorname{UIt}\left(P^{M}\left(R_{k} \cap M\right), U\right)$ be the natural elementary embedding. Now let $N=i\left(R_{k} \cap M\right), V^{*}=R_{k} \cap M$, and $\ni^{*}=\left\{\langle P, x\rangle: P \in P^{M}\left(V^{*}\right) \wedge x \in i(P)\right\}$. We will show that $\left\langle N, V^{*}, \ni^{*}\right\rangle$ satisfies $(\mathrm{A})-(\mathrm{F})$. Let us note that it is known that $\forall x \in V^{*} i(x)=x, \forall P \in P^{M}\left(V^{*}\right) P=i(P) \cap V^{*}$, and $P^{M}\left(V^{*}\right)=P^{N}\left(V^{*}\right)$. Clearly axioms (A), (E) and (F) hold. Axiom (B) holds since, for $x \in V^{*}$ and $P \in P^{N}\left(V^{*}\right)$,

$$
x \in P \leftrightarrow i(x) \in i(P) \leftrightarrow x \in i(P) \leftrightarrow P \ni^{*} x .
$$

Axiom (C) follows from the following equivalent formulas:

$$
\begin{aligned}
\forall x \in V^{*}(P \ni * x & \leftrightarrow Q \ni * x), \\
\forall x \in V^{*}(x \in i(P) & \leftrightarrow x \in i(Q)), \\
i(P) \cap V^{*} & =i(Q) \cap V^{*}, \\
P & =Q .
\end{aligned}
$$

Towards showing (D), suppose $x_{1}, \cdots, x_{n} \in V^{*}$ and $\Phi$ satisfies (i)-(iv). Let $\Phi^{\prime}$ be the result of replacing instances of " $P \ni z$ " in $\Phi$ by

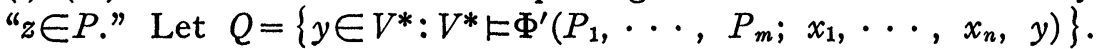
Hence

$$
\begin{aligned}
V^{*} & \vDash \forall y\left(y \in Q \leftrightarrow \Phi^{\prime}\left(P_{1}, \ldots, P_{m} ; x_{1}, \ldots, x_{n}, y\right)\right), \\
i\left(V^{*}\right) & \vDash \forall y\left(y \in i(Q) \leftrightarrow \Phi^{\prime}\left(i\left(P_{1}, \ldots, P_{m}\right) ; x_{1}, \ldots, x_{n}, y\right)\right), \\
N & \vDash \forall y\left(Q \ni y \leftrightarrow \Phi\left(P_{1}, \ldots, P_{m} ; x_{1}, \ldots, x_{n}, y\right)\right) .
\end{aligned}
$$

\section{REFERENCES}

1. R. Jensen, Some combinatorial properties of $L$ and $V$ (mimeographed).

2. K. Kunen, Some applications of iterated ultrapowers in set theory, Ann. Math. Logic 1 (1970), 179-227.

3. J. Silver, Some applications of model theory in set theory, Doctoral Dissertation, University of California, Berkeley, Calif., 1966.

State University of New York at Buffalo, Amherst, New York 14226

University of California, los Angeles, California 90024 